\title{
Surrogate Markers of Overall Survival in Metastatic Colorectal Cancer: An Evolving Challenge Still More Complex with Repeat Surgery
}

\author{
René Adam, MD, PhD \\ AP-HP Hôpital Paul Brousse, Centre Hépato-Biliaire, Inserm UMRS 776, Univ Paris-Sud, Villejuif, France
}

The gold standard end point for assessing efficacy of treatment in any cancer is overall survival (OS), but OS requires prolonged follow-up. Accordingly, efforts have been repeatedly made since the last decade to propose surrogate markers of OS sufficiently robust to be able to shorten the follow-up duration of clinical trials. Diseasefree survival (DFS) at 3 years has been probably one of the most popular in colorectal cancer ${ }^{1}$ and even more recently in gastric cancer. ${ }^{2}$ Progression-free survival has been proposed with some success in renal cancer ${ }^{3}$ but with inconsistent results in advanced gastric cancer. ${ }^{4}$ These surrogate markers are however unadapted for strategies involving surgery-particularly repeat surgery-because surgery is the only treatment able to change the condition of a patient with a tumor in place, thus, with disease, to that of a patient without any tumor and therefore disease-free. Accordingly, the traditional evaluation of cancer treatments providing that, when a recurrence event occurs in the history of the disease, the patient is definitively censored from the curve of DFS or recurrence-free survival, should be revisited in case of repeat surgery because the patient could become disease-free again.

In this article, Oba et al. have treated this important issue. In a study involving 371 patients with liver-limited metastases who underwent operation in a very experienced center over a 17-year period, they confirm the survival discrepancy between recurrence-free survival and OS in patients with resectable colorectal liver metastases and propose a new composite end point, time to surgical failure (TSF), which seems to be more strongly associated with OS and better

\section{(C) Society of Surgical Oncology 2014}

First Received: 16 January 2014;

Published Online: 8 February 2014

R. Adam, MD, PhD

e-mail: rene.adam@pbr.aphp.fr capable of reflecting the long-term outcome. This is not surprising because, conversely to recurrence-free survival or DFS, this new marker integrates the survival benefit provided by repeat hepatectomy and we know that despite the tremendous progress made by chemotherapy in recent years, surgery is still the only one able to provide the best survival benefit at long term and sometimes to give a chance of cure.

One may argue that this concerns a low proportion of patients-only 10-20\% are initially resectable-and for those who will further be selected for repeat hepatectomy, the proportion will further reduce. However, the increasing use of the Onco-Surge strategy, which combines optimal chemotherapy to downsize initially unresectable metastases, with secondary surgery, is offering more and more patients the possibility to be resected and to benefit from a prolonged survival (around $33 \%$ at 5 years; www. livermetsurvey.org). In connection, with this, when a recurrence occurs-a frequent event concerning 70-75\% of patients in the study-repeat resection is increasingly proposed by most surgical teams owing to the equivalent gain in survival compared with the first hepatectomy. One of the strengths of the study is to show that this repeat surgery is not anecdotal and that repeat resections were performed in $54.3 \%$ of first relapses, $40.7 \%$ of second relapses, and $47.1 \%$ of third relapses. If traditional primary DFS were used, 40-54\% of patients would have been underevaluated in terms of long-term outcome since censored from the primary DFS curve, while they became again disease-free after the second hepatectomy. The same will also occur for further repeat resections.

One of the limitations of the study could be, paradoxically, the high expertise in liver surgery of the authors, meaning that the high proportion of repeat hepatectomy reported in the article could not be shared by all surgical teams worldwide.

Another issue concerns the absence of any perioperative chemotherapy in the strategy proposed by the authors. 
Although the attitude of surgery up front in single metachronous metastases is defendable, ${ }^{5}$ the use of neoadjuvant chemotherapy is generally supported by a randomized trial at least in terms of 3 -year DFS. ${ }^{6}$ A validation of the reliability of the TSF in this population would be useful.

A last point concerns the potential advantage of using TSF in place of OS in clinical trials. One of the advantage of a surrogate marker, in addition to its good correlation with OS, is the earlier information that it gives with regard to further OS. No evaluation has been made in the article regarding this point, but no doubt that the authors will soon explore this aspect.

In summary, we still need valuable surrogate markers of OS, especially in patients submitted to a surgical or a oncosurgical strategy. With the increased expertise in hepatic surgery, these patients have become more and more frequent, either up front or after conversion chemotherapy. The merit of the article of Oba et al. is to propose a new surrogate marker of OS that is better than DFS in resectable patients treated up front by surgery. This marker needs now to be validated in resectable or unresectable patients receiving perioperative chemotherapy. The more the repeat surgery will be used, the less valid will be the traditional surrogacy of DFS or regression-free survival with regard to OS.

This also extends to the largest need of harmonization between evaluation criteria regarding the effects of chemotherapy, usually the median survival from the diagnosis of metastases, and those of surgery, usually median or 5-year survival from the time of resection. Because resection may occur several months after the initiation of chemotherapy, surgically treated patients have a reported survival (from the time of resection) underestimated with regard to the diagnosis of the disease and to the results of chemotherapy alone. To harmonize the evaluation, one may propose that results after surgery may consider both the survival after diagnosis and that after the surgical treatment. In this way, median survival from diagnosis could be the common tool to be used for patients exclusively treated by chemotherapy, by surgery, or by the combination of chemotherapy and surgery.

\section{REFERENCES}

1. Sargent DJ, Wieand HS, Haller DG, Gray R, Benedetti JK, Buyse $\mathrm{M}$, et al. Disease-free survival versus overall survival as a primary end point for adjuvant colon cancer studies: individual patient data from 20,898 patients on 18 randomized trials. J Clin Oncol. 2005;23:8664-70.

2. Oba K, Paoletti X, Alberts S, Bang YJ, Benedetti J, Bleiberg H, et al. Disease-free survival as a surrogate for overall survival in adjuvant trials of gastric cancer: a meta-analysis. $J$ Natl Cancer Inst. 2013;105:1600-7.

3. Halabi S, Rini B, Escudier B, Stadler WM, Small EJ. Progressionfree survival as a surrogate endpoint of overall survival in patients with metastatic renal cell carcinoma. Cancer. 2014;120:52-60.

4. Paoletti X, Oba K, Bang YJ, Bleiberg H, Boku N, Bouche O, et al. Progression-free survival as a surrogate for overall survival in advanced/recurrent gastric cancer trials: a meta-analysis. $J$ Natl Cancer Inst. 2013;105:1667-70.

5. Adam R, Bhangui P, Poston G, Mirza D, Nuzzo G, Barroso E, et al. Is perioperative chemotherapy useful for solitary, metachronous, colorectal liver metastases? Ann Surg. 2010;252:774-87.

6. Nordlinger B, Sorbye H, Glimelius B, Poston GJ, Schlag PM, Rougier $\mathrm{P}$, et al. Perioperative FOLFOX4 chemotherapy and surgery versus surgery alone for resectable liver metastases from colorectal cancer (EORTC 40983): long-term results of a randomised, controlled, phase 3 trial. Lancet Oncol. 2013;14:1208-15. 